\title{
Initiation of Guideline-Matched Oral Anticoagulant in Atrial Fibrillation-Related Stroke
}

\author{
Mi-Yeon Eun, ${ }^{\mathrm{a}}$ Jae-Young Kim, ${ }^{\text {b,c }}$ Yang-Ha Hwang, ${ }^{\mathrm{d}}$ Man-Seok Park, ${ }^{\mathrm{e}}$ Joon-Tae Kim, ${ }^{\mathrm{e}}$ Kang-Ho Choi, ${ }^{\mathrm{e}}$

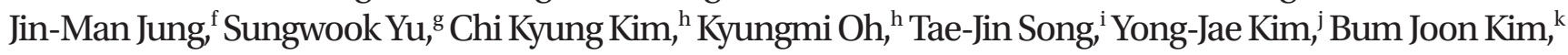 \\ Sung Hyuk Heo, ${ }^{1}$ Kwang-Yeol Park, ${ }^{\mathrm{m}}$ Jeong-Min Kim, ${ }^{\mathrm{m}}$ Jong-Ho Park, ${ }^{\mathrm{n}}$ Jay Chol Choi, ${ }^{\mathrm{o}}$ Jong-Won Chung, \\ Oh Young Bang, ${ }^{\mathrm{b}}$ Gyeong-Moon Kim, ${ }^{\mathrm{b}}$ Woo-Keun Seo ${ }^{\mathrm{b}, \mathrm{c}}$ \\ ${ }^{a}$ Department of Neurology, Kyungpook National University Chilgok Hospital, School of Medicine, Kyungpook National University, Daegu, Korea \\ ${ }^{b}$ Department of Neurology, Samsung Medical Center, Sungkyunkwan University School of Medicine, Seoul, Korea \\ 'Department of Digital Health, Samsung Advanced Institute for Health Sciences \& Technology, Sungkyunkwan University, Seoul, Korea \\ ${ }^{d}$ Department of Neurology, Kyungpook National University Hospital, School of Medicine, Kyungpook National University, Daegu, Korea \\ eDepartment of Neurology, Chonnam National University Hospital, Chonnam National University Medical School, Gwangju, Korea \\ fDepartment of Neurology, Korea University Ansan Hospital, Korea University College of Medicine, Ansan, Korea \\ ${ }^{9}$ Department of Neurology, Korea University Anam Hospital, Korea University College of Medicine, Seoul, Korea \\ hDepartment of Neurology, Korea University Guro Hospital, Korea University College of Medicine, Seoul, Korea \\ 'Department of Neurology, Ewha Womans University Seoul Hospital, Ewha Womans University School of Medicine, Seoul, Korea \\ 'Department of Neurology, Eunpyeong St. Mary's Hospital, College of Medicine, The Catholic University of Kore, Seoul, Korea \\ kDepartment of Neurology, Asan Medical Center, University of Ulsan College of Medicine, Seoul, Korea \\ 'Department of Neurology, Kyung Hee University Hospital, Kyung Hee University School of Medicine, Seoul, Korea \\ mDepartment of Neurology, Chung-Ang University Hospital, Chung-Ang University College of Medicine, Seoul, Korea \\ "Department of Neurology, Myongji Hospital, Hanyang University College of Medicine, Goyang, Korea \\ ${ }^{\circ}$ Department of Neurology, Jeju National University Hospital, Jeju National University College of Medicine, Jeju, Korea
}

Background and Purpose To evaluate the outcome events and bleeding complications of the European Society of Cardiology (ESC) guideline-matched oral anticoagulant therapy for patients with acute ischemic stroke and atrial fibrillation (AF).

Methods Patients with acute ischemic stroke and AF from a nationwide multicenter registry (Korean ATrial fibrillaTion EvaluatioN regisTry in Ischemic strOke patieNts [K-ATTENTION]) between January 2013 and December 2015 were included in the study. Patients were divided into the ESC guideline-matched and the non-matched groups. The primary outcome was recurrence of any stroke during the 90-day follow-up period. Secondary outcomes were major adverse cerebrovascular and cardiovascular events, ischemic stroke, intracranial hemorrhage, acute coronary syndrome, allcause mortality, and major hemorrhage. Propensity score matching and logistic regression analyses were performed to assess the effect of the treatments administered.

Results Among 2,321 eligible patients, 1,126 patients were 1:1 matched to the ESC guidelinematched and the non-matched groups. As compared with the non-matched group, the ESC guideline-matched group had a lower risk of any recurrent stroke (1.4\% vs. 3.4\%; odds ratio [OR], 0.41 ; $95 \%$ confidence interval [Cl], 0.18 to 0.95 ). The risk of recurrent ischemic stroke was lower in the ESC guideline-matched group than in the non-matched group (0.9\% vs. 2.7\%; $0 \mathrm{R}, 0.32 ; 95 \% \mathrm{Cl}$, 0.11 to 0.88 ). There was no significant difference in the other secondary outcomes between the two groups.

Conclusions ESC guideline-matched oral anticoagulant therapy was associated with reduced risks
Correspondence: Yang-Ha Hwang Department of Neurology, Kyungpook National University Hospital, School of Medicine, Kyungpook National University, 130 Dongdeok-ro, Jung-gu, Daegu 41944, Korea

Tel: +82-53-420-5758

Fax: +82-53-422-4265

E-mail:yangha.hwang@gmail.com https://orcid.org/0000-0002-66657481

Co-correspondence: Woo-Keun Seo Department of Neurology, Samsung Medical Center, Sungkyunkwan University School of Medicine, 81 Irwon-ro, Gangnam-gu, Seoul 06351, Korea

Tel: +82-2-3410-0799

Fax: +82-2-3410-0630

E-mail: mcastenosis@gmail.com https://orcid.org/0000-0002-40048434

Received: August 14, 2020

Revised: November 18, 2020

Accepted: November 19, 2020 
of any stroke and ischemic stroke as compared with the non-matched therapy.

Keywords Stroke; Atrial fibrillation; Guideline; Anticoagulants; Outcome

\section{Introduction}

In patients with atrial fibrillation (AF), long-term oral anticoagulant therapy effectively reduces the risk of stroke and systemic embolism, ${ }^{1,2}$ and it is the primary treatment for the prevention of stroke recurrence. AF-related stroke in the acute stage has a high probability of recurrent ischemic events and hemorrhagic transformation. ${ }^{3,4}$ Therefore, the early initiation of anticoagulant therapy after stroke onset may reduce the recurrence of ischemic events but also increase hemorrhagic complications. Currently, the appropriate time to initiate anticoagulant therapy in patients with acute ischemic stroke and AF remains unclear.

To date, there have been no randomized controlled trials assessing the appropriate time to initiate oral anticoagulant therapy in patients with AF-related stroke. Although the Acute Stroke With Xarelto to Reduce Intracranial Hemorrhage, Recurrent Embolic Stroke, and Hospital Stay (Triple AXEL) and the Dabigatran Treatment of Acute Stroke II (DATAS II) trial demonstrated that early oral anticoagulant therapy following acute ischemic stroke onset is efficient and safe, its focus was on the anticoagulation strategy and not the time to initiate oral anticoagulant therapy., ${ }^{5,6}$ The study population was limited to patients with mild stroke. Observational studies that assessed the appropriate time to initiate oral anticoagulant therapy have reported conflicting results. ${ }^{7,8}$

Clinical practice guidelines vary across countries or organizations. ${ }^{9-11}$ Among the various guidelines, the European Society of Cardiology (ESC) guidelines published in 2016 provided relatively specific guidance for initiating oral anticoagulant therapy according to stroke severity. ${ }^{10}$ However, the recommendations are based on expert consensus without high-level evidence.

Therefore, we aimed to evaluate the short-term clinical outcome events and bleeding complications of the ESC guidelinematched oral anticoagulant therapy as compared with the non-matched therapy in patients with AF-related stroke using real-world data.

\section{Methods}

\section{Study design and settings}

We conducted a retrospective study analyzing data from a pro- spective, multicenter, nationwide stroke registry: Korean ATrial fibrillaTion EvaluatioN regisTry in Ischemic strOke patieNts (KATTENTION). ${ }^{12}$ This registry comprises data of consecutive patients with $\mathrm{AF}$ and acute ischemic stroke confirmed by brain imaging (computed tomography or magnetic resonance imaging) within 7 days of symptom onset from 11 comprehensive stroke centers in South Korea between January 2013 and December 2015. The registry contains demographic data, medical history of vascular risk factors, and specific data for stroke including stroke severity and etiology, laboratory findings, brain imaging findings, and clinical outcomes during the follow-up period. Patients were followed up for at least 3 months and for as long as 4.5 years. We obtained information on the type and dosage of antithrombotic drugs administered during the hospitalization and after discharge from each center. We analyzed the effect of ESC guideline-matched anticoagulant therapy on clinical outcomes. Further details regarding the K-ATTENTION registry have been reported in a previous study. ${ }^{12}$

\section{Standard protocol approval, registration, and patient consent}

This study was approved by the Institutional Review Board at each participating center. Because the data were anonymized and the risk of this study to participants was minimal, informed consent from patients was waived.

\section{Study population}

Eligibility criteria included the following: (1) acute ischemic stroke within 7 days from onset as confirmed by relevant brain imaging; (2) AF previously or newly diagnosed with electrocardiography or 24-hour Holter monitoring after the index stroke; and (3) age $\geq 20$ years. Exclusion criteria included (1) no oral anticoagulants within 30 days of the index stroke or (2) no data on the initial National Institutes of Health Stroke Scale (NIHSS) score, which was used to determine the ESC guidelinematched oral anticoagulant therapy. The patients were divided into two groups based on the conformity to the ESC guidelines of the oral anticoagulant therapy after the diagnosis of stroke. The following participants were included in the ESC guidelinematched group: (1) patients with an initial NIHSS score of $<8$ who received anticoagulant therapy for $\geq 3$ days after the acute event; (2) patients with an initial NIHSS score between 8 and 15 who received anticoagulant therapy $\geq 6$ days after the acute 
event; and (3) patients with an initial NIHSS score of $\geq 16$ who received anticoagulant therapy $\geq 12$ days after the acute event. ${ }^{10}$ Patients who were prescribed oral anticoagulants earlier than recommended by the ESC guidelines were included in the non-matched group. The ESC guidelines recommend deciding the timing of starting oral anticoagulation (OAC) based on NIHSS score and additional clinical factors. ${ }^{10}$ The ESC guidelines recommend considering a delay in initiation of OAC in some situations, such as hemorrhagic transformation, large brain infarction on imaging, unstable neurological state, and uncontrolled hypertension. Thus, delayed anticoagulation due to hemorrhagic transformation or other possible bleedingprone conditions did not violate the guidelines. Therefore, patients who started anticoagulant therapy after the recommended day of the guidelines were included in the ESC guideline-matched group.

\section{Propensity score matching}

To reduce selection bias and the imbalance in baseline characteristics between the ESC guideline-matched and the nonmatched groups, we conducted propensity score (PS) matching. PS was estimated for each participant using a multivariable logistic regression model with covariates including age, sex, hypertension, diabetes mellitus, dyslipidemia, coronary artery disease, congestive heart failure, prior stroke or transient ischemic attack (TIA), peripheral artery disease, current smoking, prestroke OAC, prestroke modified Rankin Scale (mRS), initial NIHSS score, intravenous thrombolysis, mechanical thrombectomy, $\mathrm{CHADS}_{2}$ (congestive heart failure, hypertension, age $\geq 75$ years, diabetes mellitus, prior stroke or TIA, or thromboembolism [doubled]) score, and parenteral anticoagulation. The distribution of PS sufficiently overlapped between the nonmatched and ESC guideline-matched groups; therefore, the patients were matched 1:1 with five greedy methods using the OneToManyMTCH algorithm. ${ }^{13}$ We evaluated the overall quality of the matching using a Love plot.

\section{Outcome measurements}

The primary outcome was any stroke recurrence, including ischemic and hemorrhagic stroke, from the day of admission to 90 days after the index stroke. Secondary outcomes comprised major adverse cerebrovascular and cardiovascular events (MACCE), ischemic stroke, intracranial hemorrhage, acute coronary syndrome, all-cause mortality, and major hemorrhage during the same period. MACCE was defined as nonfatal stroke, acute coronary syndrome, major hemorrhage, and all-cause mortality within 90 days. Outcomes were ascertained in the outpatient clinic from the patients or their family members. For patients who were unable to visit the outpatient clinic, trained nurses captured outcome events by telephone interviews.

\section{Statistical analysis}

Continuous variables are presented as mean \pm standard deviation values or median values and interquartile range (IOR) as appropriate. Categorical variables are expressed as frequency and percentages. We compared the baseline characteristics between the ESC guideline-matched and the nonmatched groups using Student's t-tests or Mann-Whitney U tests and chi-square tests. After PS matching of the study population, we performed logistic regression analyses to determine the effect of ESC guideline-matched oral anticoagulant therapy on clinical outcomes. To improve the performance of the model, adjustments were conducted using variables including age, sex, hypertension, diabetes mellitus, dyslipidemia, coronary artery disease, congestive heart failure, prior stroke or TIA, peripheral artery disease, current smoking, prestroke mRS, initial NIHSS, intravenous thrombolysis, mechanical thrombectomy, $\mathrm{CHADS}_{2}$ score, parenteral anticoagulation, and the type of poststroke oral anticoagulants administered. In addition, we conducted stratified Cox regression analysis considering competing risk. Kaplan-Meier estimates of the cumulative outcome events were calculated, and the event rates were compared using the log-rank test. Using multivariable logistic regression analyses, we performed subgroup analyses with predefined variables with the assessment of the interaction term. Two-sided $P$ values of $<0.05$ were considered statistically significant. All analyses were performed using SAS software version 9.4 (SAS Institute, Cary, NC, USA).

\section{Results}

\section{Study population}

Overall, 3,213 patients with acute stroke and AF between January 2013 and December 2015 were enrolled in the K-ATTENTION registry. After excluding 877 patients because of no oral anticoagulant therapy within 30 days and 15 patients without initial NIHSS scores, we analyzed the data of 2,321 patients (mean age, 72.8 years; $53.2 \%$ men). As compared with the excluded patients, the included patients were more likely to have dyslipidemia (25.7\% vs. $19.2 \%)$, to have received prestroke OAC therapy (28.4\% vs. $21.9 \%)$, and to have lower initial $\mathrm{NI}-$ HSS ( 6 vs. 13), and CHADS$_{2}$ scores ( 3 vs. 4 ) and less likely to have received intravenous thrombolysis (20.3\% vs. $27.8 \%$ ) (Supplementary Table 1). Of these patients, 1,737 were assigned to the non-matched group (mean age, 73.1 years; $52.0 \%$ men), and 584 patients were assigned to the ESC 


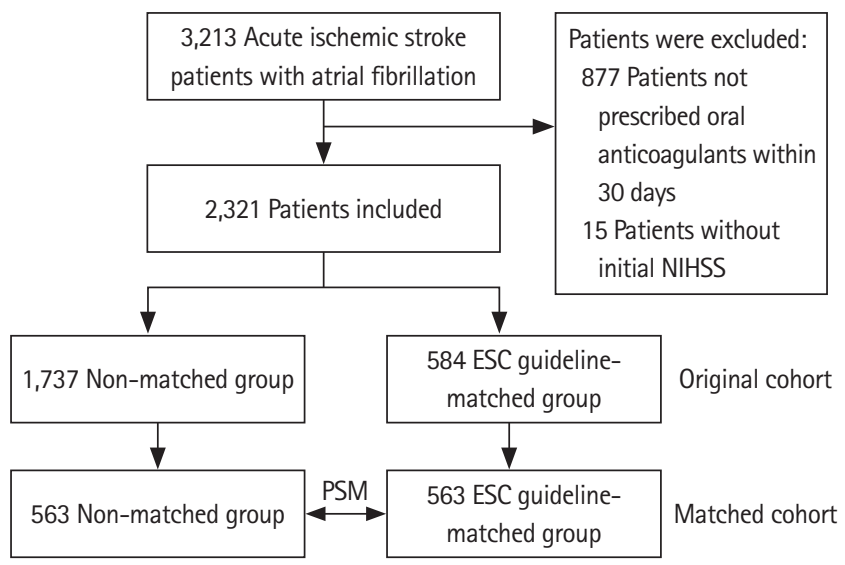

Figure 1. Selection of the study population. NIHSS, National Institutes of Health Stroke Scale; ESC, European Society of Cardiology; PSM, propensity score matching. guideline-matched group (mean age, 71.9 years; 56.7\% men) (Figure 1).

As compared with the ESC guideline-matched group, the non-matched group was older and had more severe neurological deficits at baseline (median NIHSS score, 8 [IOR, 2 to 15] vs. 4 [IOR, 1 to 9], $P<0.001$ ), a higher $\mathrm{CHADS}_{2}$ score (3 vs. 3 , $P=0.046)$, and a more frequent history of prior stroke or TIA (36.1\% vs. $28.8 \%, P=0.001$ ) (Table 1). Mechanical thrombectomy was performed in $11.9 \%$ of patients in the non-matched group and $7.0 \%$ of those in the ESC guideline-matched group $(P=0.001)$. The median time to initiate anticoagulant therapy was 0 day (IQR, 0 to 2$)$ in the non-matched group and 6 day $(I Q R$, 4 to 13$)$ in the ESC guideline-matched group. The proportion of patients receiving warfarin and non-vitamin $\mathrm{K}$ an-

Table 1. Baseline characteristics of participants before and after propensity score matching

\begin{tabular}{|c|c|c|c|c|c|c|}
\hline \multirow[b]{2}{*}{ Characteristic } & \multicolumn{3}{|c|}{ Original cohort } & \multicolumn{3}{|c|}{ Matched cohort ${ }^{+}$} \\
\hline & $\begin{array}{l}\text { Non-matched } \\
(n=1,737)\end{array}$ & $\begin{array}{l}\text { Guideline-matched } \\
\qquad(\mathrm{n}=584)\end{array}$ & $P^{*}$ & $\begin{array}{c}\text { Non-matched } \\
(n=563)\end{array}$ & $\begin{array}{l}\text { Guideline-matched } \\
\qquad(\mathrm{n}=563)\end{array}$ & $P^{*}$ \\
\hline Age (yr) & $73.1 \pm 9.9$ & $71.9 \pm 10.0$ & 0.003 & $72.8 \pm 10.2$ & $71.9 \pm 10.0$ & 0.040 \\
\hline Male sex & $903(52.0)$ & $331(56.7)$ & 0.049 & $312(55.4)$ & $322(57.2)$ & 0.548 \\
\hline \multicolumn{7}{|l|}{ History of risk factors } \\
\hline Hypertension & $1,195(68.8)$ & $399(68.3)$ & 0.831 & $373(66.3)$ & $382(67.9)$ & 0.568 \\
\hline Diabetes mellitus & $475(27.3)$ & $155(26.5)$ & 0.705 & $137(24.3)$ & $146(25.9)$ & 0.536 \\
\hline Dyslipidemia & $455(26.2)$ & $142(24.3)$ & 0.369 & $142(25.2)$ & $134(23.8)$ & 0.579 \\
\hline Coronary artery disease & $228(13.1)$ & $73(12.5)$ & 0.697 & $65(11.5)$ & $68(12.1)$ & 0.782 \\
\hline Congestive heart failure & $82(4.7)$ & $17(2.9)$ & 0.061 & $14(2.5)$ & $13(2.3)$ & 0.846 \\
\hline Prior stroke or TIA & $627(36.1)$ & $168(28.8)$ & 0.001 & $161(28.6)$ & $183(32.5)$ & 0.155 \\
\hline Peripheral artery disease & $226(13.0)$ & 93 (15.9) & 0.617 & $6(1.1)$ & $9(1.6)$ & 0.436 \\
\hline Current smoking & $1,195(68.8)$ & $399(68.3)$ & 0.077 & $80(14.2)$ & $91(16.2)$ & 0.361 \\
\hline Prestroke OAC & $536(30.9)$ & $123(21.1)$ & $<0.001$ & $123(21.8)$ & $123(21.8)$ & 1.000 \\
\hline Prestroke mRS & $0(0-1)$ & $0(0-1)$ & 0.001 & $0(0-1)$ & $0(0-1)$ & 0.107 \\
\hline Initial NIHSS & $8(2-15)$ & $4(1-9)$ & $<0.001$ & $4(1-9)$ & $4(1-9)$ & 1.000 \\
\hline Intravenous thrombolysis & $360(20.7)$ & $112(19.2)$ & 0.422 & $105(18.7)$ & $108(19.2)$ & 0.819 \\
\hline Mechanical thrombectomy & 207 (11.9) & $41(7.0)$ & 0.001 & $33(5.9)$ & $36(6.4)$ & 0.709 \\
\hline $\mathrm{CHADS}_{2}$ score & $3(3-4)$ & $3(3-4)$ & 0.046 & $3(3-4)$ & $3(3-4)$ & 0.711 \\
\hline Parenteral anticoagulation & $448(25.8)$ & $152(26.0)$ & 0.913 & $157(27.9)$ & $145(25.8)$ & 0.459 \\
\hline Acute antiplatelet therapy & $789(45.4)$ & $336(57.5)$ & $<0.001$ & $258(45.8)$ & $324(57.5)$ & $<0.001$ \\
\hline Days to OAC therapy & $0(0-2)$ & $6(4-13)$ & $<0.001$ & $0(0-1)$ & $6(4-13)$ & $<0.001$ \\
\hline $\begin{array}{l}\text { Hemorrhagic transformation } \\
\text { (original cohort, } n=1,906 \text {; matched cohort, } n=564 \text { ) }\end{array}$ & $199(11.5)$ & 89 (15.2) & $<0.001$ & $58(10.3)$ & $85(15.1)$ & 0.002 \\
\hline Poststroke OAC & & & $<0.001$ & & & $<0.001$ \\
\hline Warfarin & 1,626 (93.6) & $486(83.2)$ & & $524(93.1)$ & 466 (82.8) & \\
\hline NOAC & $111(6.4)$ & $98(16.8)$ & & $39(6.9)$ & 97 (17.2) & \\
\hline
\end{tabular}

Values are presented as mean \pm standard deviation, number $(\%)$, or median (interquartile range).

TIA, transient ischemic attack; OAC, oral anticoagulation; mRS, modified Rankin Scale; NIHSS, National Institutes of Health Stroke Scale; CHADS $_{2}$, congestive heart failure, hypertension, age $\geq 75$ years, diabetes mellitus, prior stroke or TIA, or thromboembolism (doubled); NOAC, non-vitamin K antagonist oral anticoagulant.

${ }^{*}$ Continuous variables were compared between groups using Student's t-tests or Mann-Whitney U tests. The chi-square test was used for noncontinuous variables; ${ }^{+}$Propensity score matching was performed using a logistic regression model with covariates including age, sex, hypertension, diabetes mellitus, dyslipidemia, coronary artery disease, congestive heart failure, prior stroke or TIA, peripheral artery disease, current smoking, prestroke OAC, prestroke mRS, initial NIHSS score, intravenous thrombolysis, mechanical thrombectomy, $\mathrm{CHADS}_{2}$ score, and parenteral anticoagulation. 
Table 2. Primary and secondary outcomes within 90 days

\begin{tabular}{|c|c|c|c|c|c|c|}
\hline \multirow[b]{2}{*}{ Outcomes } & \multicolumn{3}{|c|}{ Original cohort } & \multicolumn{3}{|c|}{ Matched cohort } \\
\hline & $\begin{array}{l}\text { Non-matched } \\
(n=1,737)\end{array}$ & $\begin{array}{l}\text { Guideline-matched } \\
\qquad(n=584)\end{array}$ & $P^{*}$ & $\begin{array}{c}\text { Non-matched } \\
(n=563)\end{array}$ & $\begin{array}{l}\text { Guideline-matched } \\
\qquad(n=563)\end{array}$ & $P^{*}$ \\
\hline Any stroke & $47(2.7)$ & $8(1.4)$ & 0.066 & $19(3.4)$ & $8(1.4)$ & 0.032 \\
\hline MACCE & $156(9.0)$ & $38(6.5)$ & 0.069 & $38(6.7)$ & $37(6.6)$ & 0.905 \\
\hline Ischemic stroke & $36(2.1)$ & $5(0.9)$ & 0.054 & $15(2.7)$ & $5(0.9)$ & 0.024 \\
\hline Intracranial hemorrhage & $14(0.8)$ & $3(0.5)$ & 0.474 & $5(0.9)$ & $3(0.5)$ & 0.478 \\
\hline Acute coronary syndrome & $16(0.9)$ & $5(0.9)$ & 0.886 & $2(0.4)$ & $5(0.9)$ & 0.255 \\
\hline All-cause mortality & $93(5.4)$ & $18(3.1)$ & 0.026 & $16(2.8)$ & $17(3.0)$ & 0.860 \\
\hline Major hemorrhage & $15(0.9)$ & $10(1.7)$ & 0.086 & $5(0.9)$ & $10(1.8)$ & 0.194 \\
\hline
\end{tabular}

Values are presented as number (\%).

MACCE, major adverse cerebrovascular, and cardiovascular events.

*The chi-square test was used for noncontinuous variables.

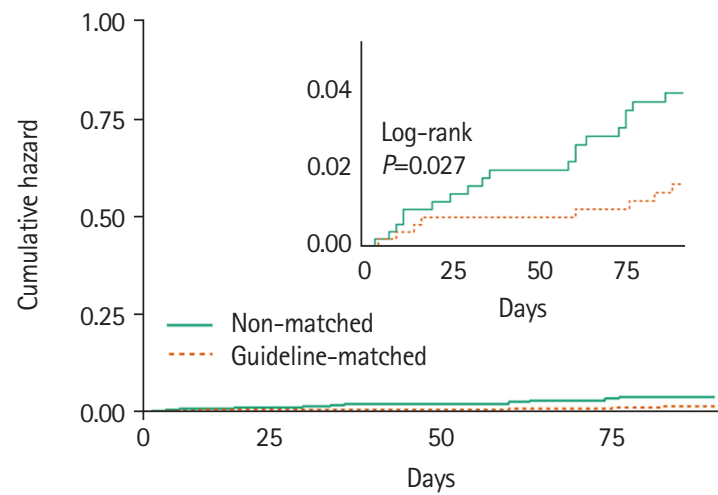

Number at risk

Non-matched 563

Guideline-matched 563

513

534

482

506

462

Figure 2. Cumulative incidence of primary outcome. The cumulative incidence of any stroke in the European Society of Cardiology guidelinematched group and the non-matched group is shown. The inset graph reveals the same data on an enlarged $y$-axis.

tagonist oral anticoagulants (NOACs) after the index stroke was $93.6 \%$ and $6.4 \%$ in the non-matched group and $83.2 \%$ and $16.8 \%$ in the ESC guideline-matched group, respectively (Table 1). Among 584 patients in the ESC guideline-matched group, 299 patients (51.2\%) started OAC within 2 days after the ESC guideline-recommended day.

PS matching analyses allocated 563 pairs of patients, and the baseline characteristics included in the PS matching were well balanced without significant differences between the non-matched and ESC guideline-matched groups (Table 1).

\section{Primary and secondary outcomes}

Among the overall cohort of 2,321 patients, 55 (2.4\%) experienced the recurrence of any stroke within 90 days. MACCE and ischemic stroke occurred in 194 patients (8.4\%) and 41 patients (1.8\%), respectively. A total of 111 patients (4.8\%) died within
90 days of the index stroke. The recurrence of any stroke in the ESC guideline-matched group (1.4\%) was lower than that in the non-matched group (2.7\%), but the difference was not significant. The ESC guideline-matched group had a lower rate of all-cause mortality within 90 days of hospitalization (3.1\% vs. $5.4 \%, P=0.026$ ) than did the non-matched group (Table 2). The ESC guideline-matched group tended to have a lower risk of MACCE (6.5\% vs. 9.0\%, $P=0.069)$ and ischemic stroke ( $0.9 \%$ vs. $2.1 \%, P=0.054)$ than did the non-matched group.

In the PS-matched cohort, the recurrence of any stroke was low in the ESC guideline-matched group than in the nonmatched group (1.4\% vs. 3.4\%, $P=0.032$ ) (Table 2 and Figure 2). There were fewer recurrent ischemic strokes in the ESC guideline-matched group (0.9\% vs. 2.7\%, $P=0.024)$ as compared with the non-matched group. MACCE occurred in $6.6 \%$ of the patients in the ESC guideline-matched group and in $6.7 \%$ of those in the non-matched group $(P=0.905)$. Supplementary Table 2 shows the event rates according to the timing of initiation of OAC related to the ESC guideline recommendation (before the guideline-recommended day; within 2 days of the guidelinerecommended day; after 2 days of the guideline-recommended day). In the ESC guideline-matched group, 289 patients (51.3\%) started oral anticoagulants within 2 days of the ESC guidelinerecommended day. Outcome events were similar between administration of OAC within 2 days and OAC after 2 days of the ESC guideline-recommended day. The $\mathrm{CHADS}_{2}$ score was also comparable between groups (OAC within 2 days of the guideline-recommended day, $3.3 \pm 0.9 ;$ OAC after 2 days of the guideline-recommended day, $3.4 \pm 0.9 ; P=0.37$ ).

\section{Effect of OAC}

In the PS-matched cohort, the ESC guideline-matched group had a lower recurrence of any stroke (odds ratio [OR], 0.41; 
Table 3. Effect of ESC guideline-matched oral anticoagulant therapy on clinical outcomes before and after propensity score matching

\begin{tabular}{|c|c|c|c|c|}
\hline Outcome & Univariate analysis & $P^{*}$ & Multivariate analysis & $P^{*}$ \\
\hline \multicolumn{5}{|l|}{ Original cohort } \\
\hline Any stroke & $0.50(0.24-1.06)$ & 0.072 & $0.50(0.24-1.06)$ & 0.072 \\
\hline MACCE & $0.71(0.49-1.02)$ & 0.063 & $0.84(0.57-1.22)$ & 0.347 \\
\hline Ischemic stroke & $0.41(0.16-1.05)$ & 0.062 & $0.36(0.14-0.92)$ & 0.032 \\
\hline Intracranial hemorrhage & $0.64(0.18-2.22)$ & 0.477 & $0.67(0.19-2.36)$ & 0.537 \\
\hline Acute coronary syndrome & $0.93(0.34-2.55)$ & 0.886 & $0.87(0.32-2.39)$ & 0.785 \\
\hline All-cause mortality & $0.56(0.34-0.94)$ & 0.028 & $0.79(0.47-1.35)$ & 0.388 \\
\hline Major hemorrhage & $2.00(0.89-4.48)$ & 0.092 & $1.56(0.68-3.60)$ & 0.294 \\
\hline \multicolumn{5}{|l|}{ Matched cohort } \\
\hline Any stroke & $0.41(0.18-0.95)$ & 0.038 & $0.41(0.18-0.95)$ & 0.038 \\
\hline MACCE & $0.97(0.61-1.55)$ & 0.941 & $0.97(0.61-1.56)$ & 0.904 \\
\hline Ischemic stroke & $0.33(0.12-0.91)$ & 0.032 & $0.32(0.11-0.88)$ & 0.028 \\
\hline Intracranial hemorrhage & $0.60(0.14-2.51)$ & 0.483 & $0.60(0.14-2.51)$ & 0.483 \\
\hline Acute coronary syndrome & $2.51(0.49-13.01)$ & 0.272 & $2.38(0.44-13.00)$ & 0.316 \\
\hline All-cause mortality & $1.06(0.53-2.13)$ & 0.860 & $1.09(0.53-2.23)$ & 0.814 \\
\hline Major hemorrhage & $2.02(0.69-5.94)$ & 0.203 & $1.47(0.48-4.48)$ & 0.499 \\
\hline
\end{tabular}

Values are presented as odds ratios and 95\% confidence interval. In the original cohort, MACCE was adjusted by age, congestive heart failure, prestroke modified Rankin Scale (mRS), CHADS2 (congestive heart failure, hypertension, age $\geq 75$ years, diabetes mellitus, prior stroke or transient ischemic attack [TIA], or thromboembolism [doubled]) score, and initial National Institutes of Health Stroke Scale (NIHSS). Ischemic stroke was adjusted by diabetes mellitus and initial NIHSS. Intracranial hemorrhage was adjusted by prior stroke or TIA. Acute coronary syndrome was adjusted by age. All-cause mortality was adjusted by age, congestive heart failure, prestroke mRS, CHADS, score, and initial NIHSS. Major hemorrhage was adjusted by the type of oral anticoagulants. Major hemorrhage was adjusted by the type of poststroke oral anticoagulants. In the matched cohort, MACCE was adjusted by initial NIHSS. Ischemic stroke was adjusted by diabetes mellitus. Acute coronary syndrome was adjusted by age, current smoking, and $\mathrm{CHADS}_{2}$ score. All-cause mortality was adjusted by age, dyslipidemia, congestive heart failure, $\mathrm{CHADS}_{2}$ score, and initial NIHSS. Major hemorrhage was adjusted by the type of poststroke oral anticoagulants.

ESC, European Society of Cardiology; MACCE, major adverse cerebrovascular, and cardiovascular events.

*Logistic regression analyses were used.

95\% confidence interval [Cl], 0.18 to 0.95) (Table 3). Because of the balanced covariates, adjustment with predefined variables did not affect the results. After adjustment for diabetes mellitus, the ESC guideline-matched group also had a reduced risk of ischemic stroke $(\mathrm{OR}, 0.32 ; 95 \% \mathrm{Cl}, 0.11$ to 0.88$)$. However, the effects of ESC guideline-matched OAC on the risk of MACCE, acute coronary syndrome, all-cause mortality, and major hemorrhage were not significant. Stratified Cox regression analysis considering competing risk also demonstrated that the ESC guideline-matched group was associated with reduced risks of any recurrent stroke (hazard ratio [HR], 0.33; $95 \% \mathrm{Cl}, 0.16$ to 0.69$)$ and recurrent ischemic stroke $(\mathrm{HR}, 0.27$; $95 \% \mathrm{Cl}, 0.11$ to 0.66 ). The effects of ESC guideline-matched OAC on outcomes in the original cohort showed generally similar trends to those seen in the PS-matched cohort (Table 3).

\section{Subgroup analyses}

Figure 3 shows the results of the subgroup analyses in the PSmatched cohort. Generally, the treatment effects were consistent across the subgroups, except for hemorrhagic transformation. Of note, in patients who did not have hemorrhagic trans- formation, the ESC guideline-matched group was associated with a lower risk of any stroke compared to the non-matched group; however, the interaction was not significant. The prestroke OAC, time to initiate anticoagulant therapy ( $<5$ days vs. $\geq 5$ days), acute antiplatelet therapy, and acute parenteral anticoagulation therapy after stroke did not affect the results. Regarding the type of oral anticoagulants, ESC guideline-matched OAC was associated with a lower risk of any stroke $(O R, 0.41$; $95 \% \mathrm{Cl}, 0.17$ to 0.97$)$ and ischemic stroke $(O \mathrm{R}, 0.29 ; 95 \% \mathrm{Cl}$, 0.10 to 0.89 ) in the warfarin group. In the subgroup of NOACs, only one event of any stroke occurred in the ESC guidelinematched group (among 97 patients), and no event occurred in the non-matched group (among 39 patients).

\section{Discussion}

In the present study, the ESC guideline-matched anticoagulant therapy was associated with reduced risks of any recurrent stroke by $59 \%$ and recurrent ischemic stroke by $68 \%$ as compared with the non-matched group in patients with acute stroke and AF. The risks of MACCE, intracranial hemorrhage, 


\begin{tabular}{|c|c|c|c|c|c|c|c|}
\hline Subgroup & No. of patients & Non-matched & Guideline-matched & Odds ratio & & $95 \% \mathrm{Cl}$ & $P$-value \\
\hline Age & & & & & & & 0.243 \\
\hline$<75 \mathrm{yr}$ & 610 & $293(52.0)$ & $317(56.3)$ & $\longmapsto$ & & $0.25(0.07-0.89)$ & \\
\hline$\geq 75 \mathrm{yr}$ & 516 & $270(48.0)$ & $246(43.7)$ & $\longmapsto$ & 1 & $0.68(0.22-2.11)$ & \\
\hline Sex & & & & & & & 0.696 \\
\hline Female & 492 & $251(44.6)$ & $241(42.8)$ & $\longmapsto$ & H & $0.34(0.09-1.27)$ & \\
\hline Male & 634 & $312(55.4)$ & $322(57.2)$ & $\longmapsto$ & - & $0.48(0.16-1.41)$ & \\
\hline Hypertension & & & & & & & 0.792 \\
\hline No & 371 & 190 (33.8) & $181(32.2)$ & $\longrightarrow$ & $\longrightarrow$ & $0.34(0.07-1.72)$ & \\
\hline Yes & 755 & $373(66.3)$ & 382 (67.9) & $\longmapsto$ & -1 & $0.44(0.17-1.18)$ & \\
\hline Diabetes mellitus & & & & & & & 0.091 \\
\hline No & 843 & 426 (75.7) & 417 (74.1) & $\longmapsto$ & +1 & $0.71(0.27-1.88)$ & \\
\hline Yes & 283 & $137(24.3)$ & $146(25.9)$ & $\longmapsto$ & & $0.10(0.01-0.79)$ & \\
\hline Dyslipidemia & & & & & & & 0.175 \\
\hline No & 850 & $421(74.8)$ & 429 (76.2) & $\longmapsto$ & -1 & $0.62(0.24-1.61)$ & \\
\hline Yes & 276 & $142(25.2)$ & 134 (23.8) & $\longmapsto$ & & $0.13(0.02-1.02)$ & \\
\hline Coronary artery disease & & & & & & & 0.911 \\
\hline No & 993 & 498 (88.5) & 495 (87.9) & $\longmapsto$ & & 0.41 (0.17-0.99) & \\
\hline Yes & 133 & 65 (11.6) & $68(12.1)$ & $\square$ & - & $0.47(0.04-5.31)$ & \\
\hline Congestive heart failure & & & & & & & 0.998 \\
\hline No & 1,099 & $549(97.5)$ & $550(97.7)$ & $\longmapsto$ & & $0.41(0.18-0.95)$ & \\
\hline Yes & 27 & $14(2.5)$ & $13(2.3)$ & & & & \\
\hline Prior stroke or TIA & & & & & & & 0.333 \\
\hline No & 782 & $402(71.4)$ & $380(67.5)$ & $\longrightarrow$ & & $0.30(0.10-0.90)$ & \\
\hline Yes & 344 & $161(28.6)$ & $183(32.5)$ & $\longmapsto$ & -4 & $0.70(0.18-2.64)$ & \\
\hline Current smoking & & & & & & & 0.357 \\
\hline No & 955 & $483(85.8)$ & 472 (83.8) & $\longmapsto$ & $H$ & $0.50(0.20-1.26)$ & \\
\hline Yes & 171 & $80(14.2)$ & $91(16.2)$ & & $\dashv$ & $0.17(0.02-1.46)$ & \\
\hline $\mathrm{CHADS}_{2}$ score & & & & & & & 0.606 \\
\hline$<4$ & 620 & $302(53.6)$ & $318(56.5)$ & $\longmapsto$ & $\longrightarrow$ & $0.54(0.16-1.85)$ & \\
\hline$\geq 4$ & 506 & $261(46.4)$ & $245(43.5)$ & $\longmapsto$ & -1 & $0.34(0.11-1.08)$ & \\
\hline Pre-stroke OAC & & & & & & & 0.377 \\
\hline No & 880 & $440(78.2)$ & $440(78.2)$ & $\longmapsto$ & & $0.33(0.12-0.90)$ & \\
\hline Yes & 246 & $123(21.9)$ & $123(21.9)$ & $\longmapsto$ & - & $0.74(0.16-3.40)$ & \\
\hline Post-stroke OAC & & & & & & & 0.977 \\
\hline Warfarin & 990 & $524(93.1)$ & 466 (82.8) & $\longmapsto$ & & $0.41(0.17-0.97)$ & \\
\hline NOAC & 136 & $39(6.9)$ & $97(17.2)$ & & & & \\
\hline Days to anticoagulation & & & & & & & 0.310 \\
\hline$<5$ & 727 & 545 (96.8) & $182(32.3)$ & $\longmapsto$ & $\longrightarrow$ & $0.66(0.22-1.97)$ & \\
\hline$\geq 5$ & 399 & $18(3.2)$ & $381(67.7)$ & - & $\longrightarrow$ & $0.18(0.02-1.70)$ & \\
\hline Hemorrhagic transformation & & & & & & & 0.137 \\
\hline No & 735 & $402(87.4)$ & $333(79.7)$ & $\longrightarrow$ & & $0.27(0.08-0.96)$ & \\
\hline Yes & 399 & $58(12.6)$ & $85(20.3)$ & & - & $1.38(0.25-7.81)$ & \\
\hline Acute antiplatelet therapy & & & & & & & 0.847 \\
\hline No & 558 & $310(55.1)$ & $248(44.1)$ & $\longmapsto$ & -1 & $0.47(0.17-1.34)$ & \\
\hline Yes & 568 & $253(44.9)$ & $315(56.0)$ & $\longrightarrow$ & $\longrightarrow$ & $0.40(0.10-1.60)$ & \\
\hline Parenteral anticoagulation & & & & & & & 0.816 \\
\hline No & 814 & $406(72.1)$ & $418(74.3)$ & $\longmapsto$ & $r$ & $0.44(0.17-1.17)$ & \\
\hline Yes & 302 & $157(27.9)$ & $145(25.8)$ & $\longmapsto$ & -1 & $0.35(0.07-1.77)$ & \\
\hline Overall & & & & & & & 0.038 \\
\hline & 1,126 & $563(100.0)$ & $563(100.0)$ & $\longmapsto$ & & $0.41(0.18-0.95)$ & \\
\hline & & & & 0.20 & .002 .00 & 8.00 & \\
\hline
\end{tabular}

Figure 3. Odds ratio for the primary outcome according to the prespecified subgroups. The forest plot demonstrates the odds ratios for any stroke in the subgroups. Adjusted variables are age, sex, hypertension, diabetes mellitus, dyslipidemia, coronary artery disease, congestive heart failure, prior stroke or transient ischemic attack (TIA), peripheral artery disease, current smoking, prestroke modified Rankin Scale, initial National Institutes of Health Stroke Scale (NIHSS) score, intravenous thrombolysis, mechanical thrombectomy, and $\mathrm{CHADS}_{2}$ (congestive heart failure, hypertension, age $\geq 75$ years, diabetes mellitus, prior stroke or TIA, or thromboembolism [doubled]) score. $\mathrm{Cl}$, confidence interval; OAC, oral anticoagulation. 
all-cause mortality, and major hemorrhage were not significantly different between both groups.

Anticoagulation initiated earlier after acute ischemic stroke is likely to reduce ischemic events and to increase hemorrhagic risk. However, our study unexpectedly showed the paradoxical phenomenon that the ESC guideline-matched oral anticoagulant therapy, which was initiated later than the non-matched OAC therapy, was associated with a lower risk of ischemic stroke. The low incidence of recurrent ischemic stroke in the ESC guideline-matched group accounted for the reduced recurrence of any stroke in this group. Previous studies regarding the timing of initiating OAC therapy after acute AF-related stroke have reported heterogeneous results. In a study by Wilson et al., ${ }^{7}$ the early initiation of oral anticoagulant therapy within 4 days of AF-related stroke was not associated with lower ischemic outcomes as compared with the late initiation ( $\geq 5$ days) or absence of any oral anticoagulant therapy. Another study regarding the early and late initiation of oral anticoagulant therapy following acute stroke and AF demonstrated no significant difference in the rates of recurrent ischemic stroke and hemorrhagic stroke. ${ }^{14}$ On the other hand, the Early Recurrence and Major Bleeding in Patients With Acute Ischemic Stroke and Atrial Fibrillation Treated With Non-Vitamin K Oral Anticoagulants (RAF-NOACs) study showed that the initiation of NOACs between 3 and 14 days reduced the risk of composite outcomes compared with the early initiation within 2 days or late initiation beyond 14 days. ${ }^{8}$ Our results are in line with those of the RAF-NOACs study.

The mechanisms of the paradoxical phenomenon that more conservative initiation of $\mathrm{OAC}$ was associated with a lower risk of recurrent ischemic stroke are uncertain. A possible explanation might be the difference in antithrombotic therapies between the two groups. A recent study demonstrated that an early initiation of NOACs in patients with AF-related stroke was associated with a reduced risk of composite outcomes as compared with warfarin. ${ }^{15}$ Thus, our results might be explained by the more frequent use of NOACs in the ESC guidelinematched group than in the non-matched group (17.2\% vs. $6.9 \%)$. Although we adjusted for the type of oral anticoagulants, there may be residual confounding. Subgroup analysis regarding NOACs could not be conducted because there was no event in the non-matched group. Second, the higher proportion of acute antiplatelet therapy in the ESC guidelinematched group compared with the non-matched group (57.5\% vs. $45.8 \%$ ) might have contributed to our results. Antiplatelet therapy in patients with acute ischemic stroke reduces the incidence of recurrent ischemic stroke without a significant excess of hemorrhagic stroke. ${ }^{16}$ Because our data lack informa- tion about the timing and duration of antiplatelet therapy, further studies are needed that take into consideration these components in patients who plan OAC.

It is also possible that early anticoagulation with warfarin in the acute stage of AF-related stroke might be paradoxically associated with recurrent ischemic stroke. During this study period, warfarin was used for OAC therapy in majority of patients (93.1\% in the non-matched group and $82.8 \%$ in the guidelinematched group). Warfarin might induce a transient hypercoagulable state, particularly at the beginning of the therapy, and might increase the risk of stroke. ${ }^{17,18}$ Thrombus could be unstable in the acute stage of ischemic stroke, so that thrombus migration, growth, and embolization could occur. ${ }^{19,20}$ Thus, these paradoxical procoagulant effects of warfarin, in addition to the initial subtherapeutic international normalized ratio (INR) level, could be more significant in the acute stage of AF-related stroke. Second, early anticoagulation could promote the distal embolism of residual thrombi through thrombus dissolution. ${ }^{21,22}$ A previous study using transesophageal echocardiography reported that residual thrombus in the left atrium was detected in $43 \%$ of patients with acute thromboembolism and newly verified AF. ${ }^{23}$ The Triple AXEL study showed that about 30\% of patients who received early anticoagulation developed new ischemic lesions at 4 weeks. ${ }^{5}$ After acute AF-related stroke, unstable residual thrombus might be more vulnerable to the effects of anticoagulation. However, these inferences should be confirmed in future studies.

In terms of safety, the ESC guideline-matched oral anticoagulant therapy was not associated with a reduced risk of bleeding as compared with the non-matched oral anticoagulant therapy. However, cautious interpretation is required, because the incidences of intracranial hemorrhage $(0.5 \%$ and $0.9 \%$ in the ESC guideline-matched and non-matched groups, respectively) and major hemorrhage $(1.8 \%$ and $0.9 \%$ in the ESC guideline-matched and non-matched groups, respectively) were quite low.

In our study, the severity of stroke was likely to affect the treatment pattern of anticoagulation in AF-related stroke. The initial NIHSS score of our overall original cohort was 6 (IQR, 2 to 14), which was similar to that of previous studies (median NIHSS scores, 4 to 8). ${ }^{7,814}$ However, the initial NIHSS score was higher in the non-matched group than in the ESC guidelinematched group ( 8 vs. 4 ) before PS matching. There is the possibility that clinicians might have paid more attention and performed intensive treatment in patients with severe stroke.

Our study demonstrated the prescription pattern of oral anticoagulants in real clinical practice. In the original cohort, a large proportion of patients started oral anticoagulants earlier 
than the recommended day of the 2016 ESC guideline. In our study, we used data from a prospective, multicenter, nationwide registry between January 2013 and December 2015. Most clinicians in this study used warfarin because of the reimbursement problem of NOACs. Thus, considering the time to reach the target INR, some clinicians seemed to start warfarin earlier than the recommended day of the 2016 ESC guideline. Meanwhile, in the ESC guideline-matched group, considerable numbers of patients started OAC after the recommended day of the guideline. Paciaroni et al. ${ }^{24}$ reported that hemorrhagic transformation developed in $11 \%$ of their patients with AF-related stroke. The initiation of oral anticoagulants was delayed in these patients (mean, 23.3 days), but this delay was not associated with an increased risk of ischemic recurrence. In our study, the ESC guideline-matched group had more frequent hemorrhagic transformation than the non-matched group did (15.1\% vs. $10.3 \%$ ). Thus, our results were in line with the findings of Paciaroni et al. ${ }^{24}$

Our study has several limitations. First, although we conducted PS matching to reduce selection bias, the nonrandomized, retrospective design of this study might have a hidden selection bias and residual confounding. Second, we had only limited information about the clinical factors for the decisionmaking process of starting oral anticoagulants, such as lesion size, uncontrolled hypertension, chronic kidney disease, prior major hemorrhage, liver disease, alcohol intake, or the need for the procedure. Third, oral anticoagulants used in this study included both warfarin and NOACs. Different pharmacokinetic and pharmacodynamic profiles between warfarin and NOACs could have affected our findings. However, the results were not changed in the subgroup analysis based on the type of oral anticoagulant. Furthermore, there is limited information about clinical factors that could affect the results, including the time of AF diagnosis, drug adherence, the combination between oral anticoagulants and antiplatelet agents or parenteral anticoagulants, and change of prescription by time course. Moreover, we did not obtain information on whether the anticoagulation effect was adequately achieved, such as the time in therapeutic range of warfarin and the dose of NOACs. Therefore, a prospective study considering these factors is warranted.

Nevertheless, this study supported the usefulness of the ESC guidelines regarding the timing of initiating OAC therapy, namely, warfarin and NOACs after acute AF-related stroke. Randomized trials are ongoing, such as Optimal Timing of Anticoagulation After Acute Ischaemic Stroke (OPTIMAS) (EudraCT, 2018-003859-38), Timing of Oral Anticoagulant Therapy in Acute Ischemic Stroke With Atrial Fibrillation (TIMING) (NCT02961348), Optimal Delay Time to Initiate Anticoagulation
After Ischemic Stroke in Atrial Fibrilallation (START) (NCT03 0219 28), and Early Versus Late Initiation of Direct Oral Anticoagulants in Post-Ischaemic Stroke Patients With Atrial Fibrillation (ELAN) (NCT03148457) regarding the appropriate time to initiate NOACs. However, despite the increasing use of NOACs, warfarin is still widely used in real-world practice, ${ }^{25}$ particularly in patients with renal impairment, with mechanical valves, or who cannot afford NOACs. In addition, the ESC guidelines are the only guidelines to consider stroke severity and the risk of bleeding in detail when starting anticoagulation. In a considerable number of patients with AF-related stroke, oral anticoagulants are either not taken or used late because of a large lesion size and bleeding risk. Although stroke severity using the NIHSS score is not perfectly correlated with the lesion size, it could be a valuable and practical indicator for OAC. The findings of our study supported the ESC guideline, which guides OAC therapy in patients with severe stroke, who are likely underrepresented in clinical trials.

\section{Conclusions}

In patients with AF-related stroke, ESC guideline-matched oral anticoagulant therapy was associated with a lower recurrence of any stroke and ischemic stroke within 90 days. Further randomized studies to evaluate the appropriate time to initiate oral anticoagulant therapy in patients with AF-related acute stroke are warranted.

\section{Supplementary materials}

Supplementary materials related to this article can be found online at https://doi.org/10.5853/jos.2020.03440.

\section{Disclosure}

Woo-Keun Seo received honoraria for lectures from Pfizer, Sanofi-Aventis, Otsuka Korea, Dong-A Pharmaceutical Co. Ltd., Bayer, Daewoong Pharmaceutical Co. Ltd., Daiichi Sankyo Korea Co. Ltd., and Boryung Pharmaceutical; study grants from Daiichi Sankyo Korea Co. Ltd.; a consulting fee from OBELAB Inc.; and stock option from JLK Inspection. The others have no financial conflicts of interest.

\section{Acknowledgments}

This study was supported by the National Research Foundation of Korea and funded by the Ministry of Education (2019R1A2C 2008788). 


\section{References}

1. Hart RG, Pearce LA, Aguilar MI. Meta-analysis: antithrombotic therapy to prevent stroke in patients who have nonvalvular atrial fibrillation. Ann Intern Med 2007;146:857867.

2. Saxena R, Koudstaal P. Anticoagulants versus antiplatelet therapy for preventing stroke in patients with nonrheumatic atrial fibrillation and a history of stroke or transient ischemic attack. Cochrane Database Syst Rev 2004;4: CD000187.

3. Hart RG, Coull BM, Hart D. Early recurrent embolism associated with nonvalvular atrial fibrillation: a retrospective study. Stroke 1983;14:688-693.

4. D'Amelio M, Terruso V, Famoso G, Di Benedetto N, Realmuto $S$, Valentino $F$, et al. Early and late mortality of spontaneous hemorrhagic transformation of ischemic stroke. J Stroke Cerebrovasc Dis 2014;23:649-654.

5. Hong KS, Kwon SU, Lee SH, Lee JS, Kim YJ, Song TJ, et al. Rivaroxaban vs warfarin sodium in the ultra-early period after atrial fibrillation-related mild ischemic stroke: a randomized clinical trial. JAMA Neurol 2017;74:12061215.

6. Butcher KS, Ng K, Sheridan P, Field TS, Coutts SB, Siddiqui $M_{1}$ et al. Dabigatran treatment of acute noncardioembolic ischemic stroke. Stroke 2020;51:1190-1198.

7. Wilson $D$, Ambler $G$, Banerjee $G$, Shakeshaft $C$, Cohen $H$, Yousry TA, et al. Early versus late anticoagulation for ischaemic stroke associated with atrial fibrillation: multicentre cohort study. J Neurol Neurosurg Psychiatry 2019;90: 320-325.

8. Paciaroni M, Agnelli G, Falocci N, Tsivgoulis G, Vadikolias $\mathrm{K}$, Liantinioti $\mathrm{C}$, et al. Early recurrence and major bleeding in patients with acute ischemic stroke and atrial fibrillation treated with non-vitamin-k oral anticoagulants (RAF-NOACs) Study. J Am Heart Assoc 2017;6:e007034.

9. January CT, Wann LS, Alpert JS, Calkins H, Cigarroa JE, Cleveland JC Jr, et al. 2014 AHA/ACC/HRS guideline for the management of patients with atrial fibrillation: a report of the American College of Cardiology/American Heart Association Task Force on practice guidelines and the Heart Rhythm Society. Circulation 2014;130:e199e267.

10. Kirchhof $P$, Benussi $S$, Kotecha D, Ahlsson A, Atar D, Casadei $B$, et al. 2016 ESC guidelines for the management of atrial fibrillation developed in collaboration with EACTS. Eur Heart J 2016;37:2893-2962.

11. Ahmed N, Steiner T, Caso V, Wahlgren N; ESO-KSU ses- sion participants. Recommendations from the ESO-Karolinska Stroke Update Conference, Stockholm 13-15 November 2016. Eur Stroke J 2017;2:95-102.

12. Song TJ, Baek IY, Woo HG, Kim YJ, Chang Y, Kim BJ, et al. Characteristics and factors for short-term functional outcome in stroke patients with atrial fibrillation, nationwide retrospective cohort study. Front Neurol 2019;10:1101.

13. Parsons LS. Performing a 1:N case-control match on propensity score. Proceedings of the 29th Annual SAS Users Group International Conference; 2004 May 9-12; Montreal, CA. Cary: SAS Institute; 2004.

14. Seiffge DJ, Traenka C, Polymeris A, Hert L, Peters N, Lyrer $P$, et al. Early start of DOAC after ischemic stroke: risk of intracranial hemorrhage and recurrent events. Neurology 2016;87:1856-1862.

15. Seiffge DJ, Paciaroni M, Wilson D, Koga M, Macha K, Cappellari $M$, et al. Direct oral anticoagulants versus vitamin $\mathrm{K}$ antagonists after recent ischemic stroke in patients with atrial fibrillation. Ann Neurol 2019;85:823-834.

16. International Stroke Trial Collaborative Group. The International Stroke Trial (IST): a randomised trial of aspirin, subcutaneous heparin, both, or neither among 19435 patients with acute ischaemic stroke. Lancet 1997;349: 1569-1581.

17. Freedman MD. Oral anticoagulants: pharmacodynamics, clinical indications and adverse effects. J Clin Pharmacol 1992;32:196-209.

18. Azoulay L, Dell'Aniello S, Simon $T A$, Renoux C, Suissa S. Initiation of warfarin in patients with atrial fibrillation: early effects on ischaemic strokes. Eur Heart J 2014;35: 1881-1887.

19. Alves HC, Treurniet KM, Jansen IG, Yoo AJ, Dutra BG, Zhang $G$, et al. Thrombus migration paradox in patients with acute ischemic stroke. Stroke 2019;50:3156-3163.

20. Gorog DA, Fayad ZA, Fuster V. Arterial thrombus stability: does it matter and can we detect it? J Am Coll Cardiol 2017;70:2036-2047.

21. Corrado G, Tadeo G, Beretta S, Tagliagambe LM, Manzillo $G F$, Spata $M$, et al. Atrial thrombi resolution after prolonged anticoagulation in patients with atrial fibrillation. Chest 1999;115:140-143.

22. Kaku B. Intra-cardiac thrombus resolution after anti-coagulation therapy with dabigatran in a patient with midventricular obstructive hypertrophic cardiomyopathy: a case report. J Med Case Rep 2013;7:238.

23. Manning WJ, Silverman DI, Waksmonski CA, Oettgen $P$, Douglas PS. Prevalence of residual left atrial thrombi among patients with acute thromboembolism and newly 
recognized atrial fibrillation. Arch Intern Med 1995;155:2 193-2198.

24. Paciaroni $M$, Bandini F, Agnelli G, Tsivgoulis $G$, Yaghi $S$, Furie $\mathrm{KL}$, et al. Hemorrhagic transformation in patients with acute ischemic stroke and atrial fibrillation: time to initiation of oral anticoagulant therapy and outcomes. $J$ Am Heart Assoc 2018;7:e10133.
25. Xian Y, Xu H, O'Brien EC, Shah S, Thomas L, Pencina MJ, et al. Clinical effectiveness of direct oral anticoagulants vs warfarin in older patients with atrial fibrillation and ischemic stroke: findings from the Patient-Centered Research Into Outcomes Stroke Patients Prefer and Effectiveness Research (PROSPER) Study. JAMA Neurol 2019;76:11921202. 
Supplementary Table 1. Comparison of baseline characteristics between included and excluded patients

\begin{tabular}{|c|c|c|c|c|}
\hline Characteristic & $\begin{array}{c}\text { Total patients } \\
(n=3213)\end{array}$ & $\begin{array}{l}\text { Included patients } \\
\quad(\mathrm{n}=2,321)\end{array}$ & $\begin{array}{l}\text { Excluded patients } \\
\qquad(n=892)\end{array}$ & $P^{*}$ \\
\hline Age (yr) & $73.6 \pm 9.9$ & $72.8 \pm 9.9$ & $75.7 \pm 9.5$ & $<0.001$ \\
\hline Male sex & $1,650(51.4)$ & $1,234(53.2)$ & $416(46.6)$ & 0.001 \\
\hline \multicolumn{5}{|l|}{ History of risk factors } \\
\hline Hypertension & $2,227(69.3)$ & $1,594(68.7)$ & $633(71)$ & 0.208 \\
\hline Diabetes mellitus & $856(26.6)$ & $630(27.1)$ & $226(25.3)$ & 0.299 \\
\hline Dyslipidemia & $768(23.9)$ & $597(25.7)$ & $171(19.2)$ & $<0.001$ \\
\hline Coronary artery disease & 415 (12.9) & $301(13)$ & $114(12.8)$ & 0.887 \\
\hline Congestive heart failure & $141(4.4)$ & $99(4.3)$ & $42(4.7)$ & 0.583 \\
\hline Prior stroke or TIA & $1,058(32.9)$ & $747(32.2)$ & $311(34.9)$ & 0.148 \\
\hline Peripheral artery disease & $38(1.2)$ & $31(1.3)$ & $7(0.8)$ & 0.196 \\
\hline Current smoking & $430(13.4)$ & $319(13.7)$ & $111(12.4)$ & 0.332 \\
\hline Prestroke mRS & $0(0-1)$ & $0(0-1)$ & $0(0-2)$ & $<0.001$ \\
\hline Initial NIHSS & $8(2-15)$ & $6(2-14)$ & $13(5-19)$ & $<0.001$ \\
\hline IV thrombolysis & $720(22.4)$ & $472(20.3)$ & $248(27.8)$ & $<0.001$ \\
\hline Mechanical thrombectomy & $338(10.5)$ & $248(10.7)$ & $90(10.1)$ & 0.622 \\
\hline $\mathrm{CHADS}_{2}$ score & $3(3-4)$ & $3(3-4)$ & $4(3-4)$ & $<0.001$ \\
\hline Prestroke OAC & 854 (26.6) & $659(28.4)$ & 195 (21.9) & $<0.001$ \\
\hline
\end{tabular}

Values are presented as mean \pm standard deviation, number (\%), or median (interquartile range).

TIA, transient ischemic attack; mRS, modified Rankin Scale; NIHSS, National Institutes of Health Stroke Scale; IV, intravenous; CHADS $_{2}$, congestive heart failure, hypertension, age $\geq 75$ years, diabetes mellitus, prior stroke or TIA, or thromboembolism (doubled); OAC, oral anticoagulation.

Supplementary Table 2. Primary and secondary outcomes within 90 days according to the timing of initiation of OAC related to the ESC guideline

\begin{tabular}{|c|c|c|c|c|}
\hline Variable & $\begin{array}{l}\text { OAC before recommended day } \\
\qquad(\mathrm{n}=563)\end{array}$ & $\begin{array}{l}\text { OAC within } 2 \text { days of recommended day } \\
\qquad(n=289)\end{array}$ & $\begin{array}{l}\text { OAC after } 2 \text { days of recommended day } \\
\qquad(n=274)\end{array}$ & $P^{*}$ \\
\hline Any stroke & $19(3.4)$ & $5(1.7)$ & $3(1.1)$ & 0.725 \\
\hline MACCE & $38(6.7)$ & $20(6.9)$ & $17(6.2)$ & 0.865 \\
\hline Ischemic stroke & $15(2.7)$ & $2(0.7)$ & $3(1.1)$ & 0.678 \\
\hline $\mathrm{ICH}$ & $5(0.9)$ & $3(1.0)$ & $0(0.0)$ & 0.249 \\
\hline ACS & $2(0.4)$ & $3(1.0)$ & $2(0.7)$ & 1.000 \\
\hline Mortality & $16(2.8)$ & $9(3.1)$ & $8(2.9)$ & 1.000 \\
\hline Major hemorrhage & $5(0.9)$ & $6(2.1)$ & $4(1.5)$ & 0.753 \\
\hline
\end{tabular}

Values are presented as number (\%).

OAC, oral anticoagulation; ESC, European Society of Cardiology; MACCE, major adverse cerebrovascular and cardiovascular events; ICH, intracranial hemorrhage; ACS, acute coronary syndrome.

*The chi-square test was used for the comparison between the OAC within 2 days vs. after 2 days. 